\title{
Network Safety Policy Research for Analyzing Static and Dynamic Traffic Volume on the Basis of Data Mining
}

Jing $\mathrm{Xu}^{*}$

Ningbo Dahongying University, Ningbo 315175, China

\begin{abstract}
With popularization of network, higher requirement is proposed to intrusion detection system IDS for network safety consideration. The traditional electronic data processing is combined with safety auditing, which has become a necessary part of constituting integrated network safety technology at present, thus the methods as optimal matching mode and statistics, etc of intrusion detection system shall be adopted. This project shall respectively make comprehensive description to current situations of intrusion detection research via the aspects of intrusion detection research method (anomaly detection, misuse detection), intrusion detection system monitoring object (network based, host based), to comprehensively analyze the impact of intrusion detection system to system architecture. On this basis a network-based anomaly intrusion detection system NAIDS is designed to network anomaly intrusion, the association rules mining and frequent scenario mining are adopted to scan the intrusion characteristic, through static mining mode and dynamic mining mode, safety detection is conducted at single layer and domain layer, new type attack can be detected via improved NAIDS system. Next, NAIDS system performance shall be evaluated by aiming at various intrusion data. Generally speaking, the system performance can detect the rejection service attack and detection attack.
\end{abstract}

Keywords: Intrusion detection, data mining, anomaly detection, misuse detection, NAIDS system.

\section{PRINCIPLE AND METHOD ANALYSIS OF DATA MINING IN INTRUSION DETECTION}

NAIDS (network anomaly detection system) is the system prototype that can realize the proposed method in the available operation environment, the dynamic slide window is proposed at real time detection process to satisfy the real time requirement, classification engine is proposed to further reduce false report rate, decision tree algorithm is proposed to detect new type attack and improve application, and some hypothesis are proposed to above three main issues. The research method of NAIDS adopts data mining ideology and technology, the so called data mining is to mine the potential and unknown and valuable information from massive data. The data mining algorithm is adopted to extract safety related system characteristic attribute, and apply data mining technology to intrusion detection field, and create safety event classification model as per system characteristic attribute, to automatically identify safety event, and data mining based intrusion detection model can be established. This model includes a series of processes of safety event auditing data, such as data acquisition, pre-processing, characteristic variable selection, algorithm comparison, mining result processing and result visualization, etc, wherein, data mining is the key of whole process. This project deems that intrusion detection essentially belongs to data analysis process, data mining technology can mine the normal or intrusion behavior mode suspected association rule shall be acquired via line incremental mining algorithm, it is classified into unknown from a large number of auditing data. NAIDS system is composed of two operation mode stages as training and detection, during operation at detection status, current event, normal event or known attack type. In the anomaly event, if the attack type data once occurred during training stage, the attack type name can be further designated.

Data mining method is introduced into intrusion detection system to analyze the data, independent detection model is established through training, to relieve burden of detection system, and improve safety of detection system. The methods as association rule mining, frequent scenario mode mining, cluster mining, etc are mainly used for intrusion detection, in NAIDS, the usual data mining can be divided into: mining association rules, data class if canon, clustering analysis, mining sequential pattern. Also, data mining algorithm can also be divided into association analysis algorithm [1], data classification algorithm, clustering analysis algorithm [2], and sequential analysis algorithm [3, 4]. At present the association, sequence, classification and clustering type, etc are main data mining algorithm [5, 6] for intrusion detection, hereinafter these algorithms shall be briefly introduced.

\subsection{Association Analysis Algorithm}

The association rule form is $X \rightarrow Y_{[c, s]}$, herein $X \cup Y X$ $\cap Y=\Phi, s$ is support degree of, $\mathrm{c}$ is confidence coefficient of the rule and is defined as $\mathrm{SxUy} / \mathrm{Sx}$, and database knowledge association rule is to describe the impact degree among each attribute. 


\subsection{Sequence Analysis Algorithm}

The sequence analysis is used to mine the correlation among different data logs, its purpose is to seek out the big sequence in the transaction database satisfying minimum support degree designated by user, the algorithm can be divided into five steps as sequencing, big data item analysis, transformation, sequence modeling, sequence maximization, wherein, sequence modeling is the key of the algorithm.

\subsection{Classification Algorithm}

Data classification is to extract the characteristic attribute of data item in the database, the created classification model can map data item of database to any given class. Data classification processing is: acquire training data, centralized training data, analyze training data set, and create optimized classification model.

\subsection{Clustering Algorithm}

The analysis to data object set without knowing data rule is called clustering algorithm, the data object is divided into multiple classes or clusters, the object in the same cluster shall have very high similarity, the object of different clusters has very low similarity. The similarity mentioned herein is calculated subject to attribute value of description object, and it is usually measured by distance.

It is necessary to mine some attribute from auditing data to describe the data, some other shall only provide auxiliary information which means the important degree of attribute for describing data is different. The audit log of quantized data during mining process is divided into two sections by threshold of support degree and confidence degree, the problem of sharp boundary brought from such zoning shall impact performance of IDS. Therefore, Bridges adopted the combination of data mining algorithm (association rule mining algorithm and frequent scenario mining algorithm) and fuzzy logic to develop IDS. The attack behavior of multi level approximate mining to some application layer can be completed at one time connection, since its support degree is not higher than given threshold, it is very hard to detect such attack via association rule and frequent scenario rule.
Nevertheless, if low support degree is adopted during mining process, related mode acquired shall be a large number of service types with high frequency, Lee and Stolfo [7] propose multi level approximate mining technology to resolve this problem. The labelled data can be established by simulating intrusion behavior, the shortcoming is that it can only simulate the attack behavior of known [8] type, therefore Eskin, etc proposed a non-monitoring anomaly detection algorithm, to adopt distance measure (k-center point algorithm) to cluster the data instance into cluster set, if the data is clustered, some small cluster shall be marked as anomaly instance.

\section{OPERATION PRINCIPLE AND SYSTEM ARCHITECTURE OF MAIDS}

MAIDS is the anomaly detection system by aiming at tcpdump data, the training data without attack type data in a given protected network environment is called pure data, which is used to establish network or used for user normal behavior profile archive (Normal behavior Prof 1e). The profile archive of network behavior mode is to establish an association database rule, to mine the pre-processing network connection log and seek out the suspected mode in the profile archive and acquire suspected association rule and classify them.

The work mode of MAIDS system is divided into two stages as training and detection, the training data of training stage shall adopt normal network connection log as main part mixed by some marked attack type data, the training stage is shown in Fig. (1), detection stage in Fig. (2), Fig. (3) is the operation process of system.

The training stage is composed of modelling period classification engine period. Before the system is and training classification engine period. Normal used to detect intrusion, the training stage shall only behavior profile archive is established at modelling be executed by one time. period; classifier is established at training classification engine period. Before the system is used to detect intrusion, the training stage shall only be executed by one time.

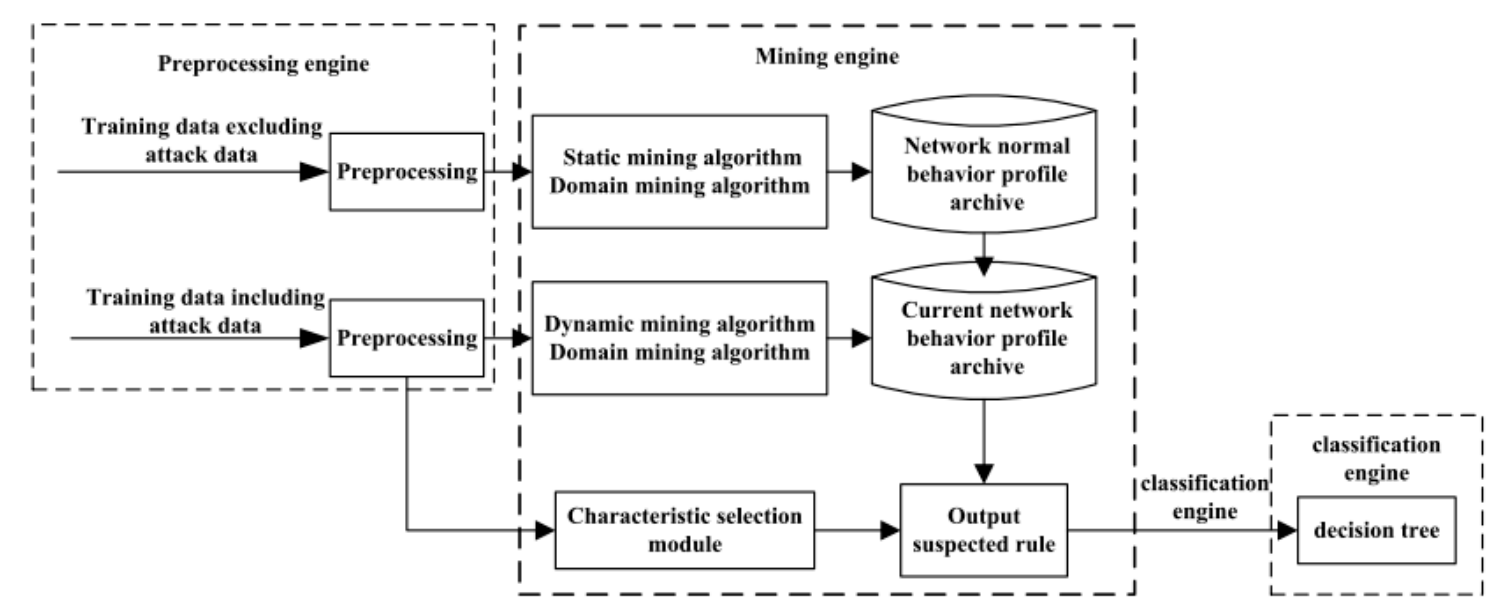

Fig. (1). MAIDS training stage. 


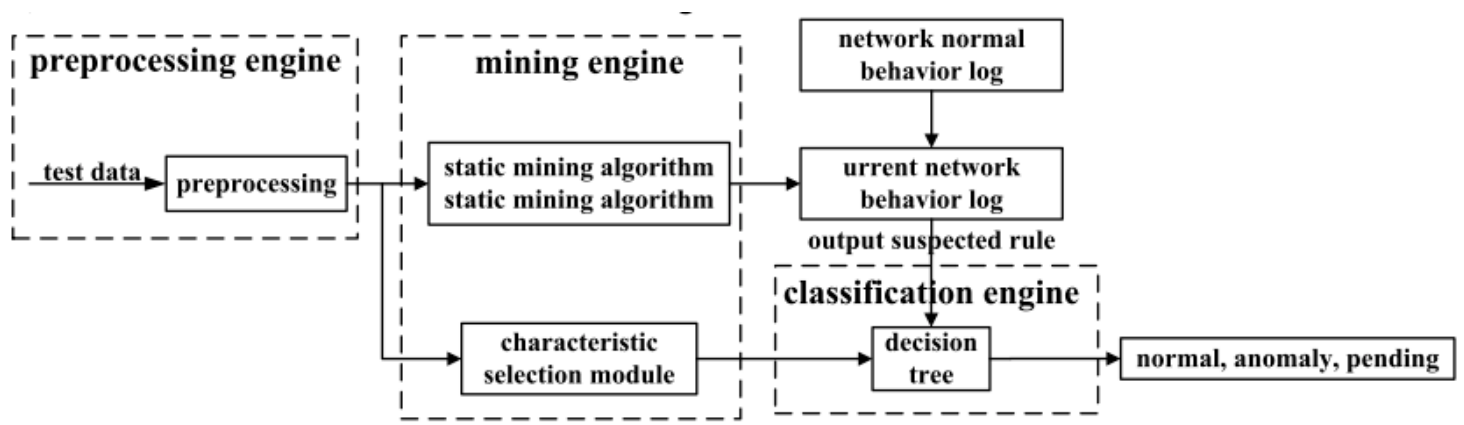

Fig. (2). Detection stage of MAIDS.

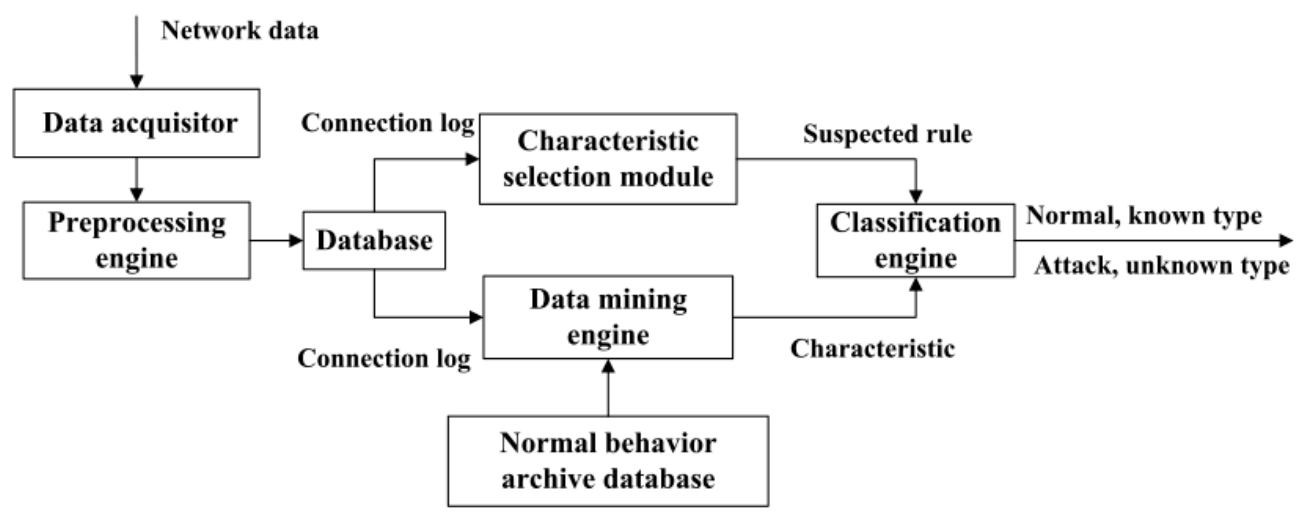

Fig. (3). NAIDS system operation process.

At detection stage, normal behavior profile archive has been acquired at training stage, and classification engine has been trained well, the real time analysis network connection $\log$ is adopted to seek out the rule set which is not contained in normal profile archive or has fairly great deviation with normal one. In classification engine of stage 1, the characteristic extracted by characteristic selection module is inputted to further divide suspected rule into normal, anomaly and unknown event. If such data type in anomaly event has occurred at classification and engine training period of training stage, it can be marked by the name of next attack type. For normal event they shall be filtered from suspected rule set without transmission to system safety staff.
The classifier of unknown event cannot at first judge it as unknown type attack, in order to minimize missing report rate, it is deemed as unknown type attack and contained in the suspected rule set, and transmitted to system safety staff for analysis purpose.

NAIDS system is generally composed of three engines as pre-processing, mining and classification, in Fig. (1), Fig. (2), the position of each engine in system operation stage is marked by dotted line box, each function engine can all be presented in some extent at two stages: Update mechanism of normal behavior profile archive is shown in Fig. (4):

Similarity higher than threshold, normal behavior without update

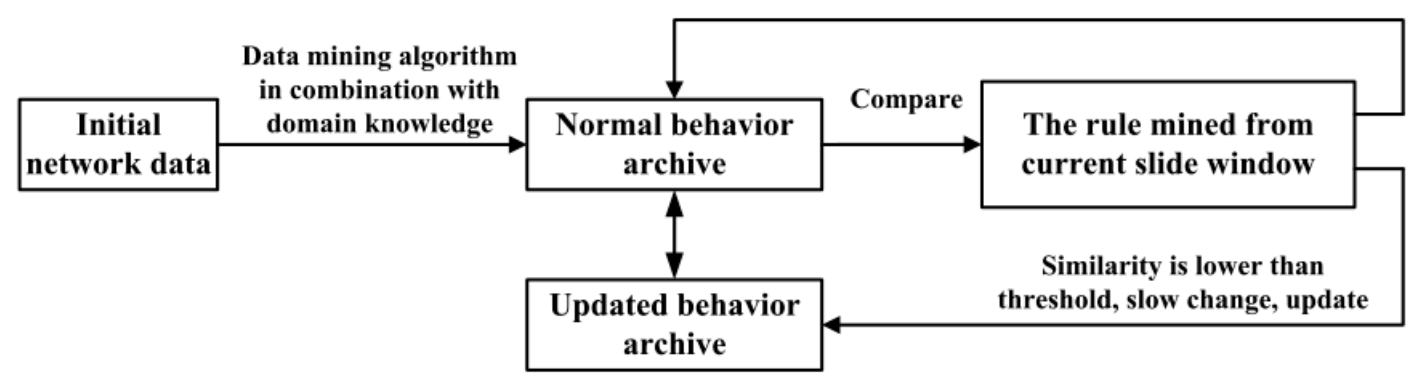

Fig. (4). Update mechanism of normal behavior profile archive. 
The slide time window method is adopted to update benchmark behavior profile archive and resolve the auditing data of long time archive and mine the data. The support degree of certain frequent item set shall decrease with time past, while support degree of certain item set in the negative margin shall gradually increase. Discarding some frequent item set and containing new item set shall exclude some data $\log$ which is deemed as out of data from the slide window. The normal behavior profile archive self adaptability frame proposed for above behavior profile archive update time and update mechanism ideology is shown in Fig. (4).

\section{NAIDS SYSTEM METHOD RESEARCH AND TEST DATA ANALYSIS}

Hereinafter two mining modes and integrated realization of two levels mining NAIDS system shall be provided. Static mining and dynamic mining mode are two mining modes; single layer and domain layer mining are two mining layers. The detailed realization algorithm of core module is presented by false code, detailed test shall be done at DARPA 1998. DARPA 1999 intrusion detection evaluation data set.

The static mining is used to seek out the rule set with its support degree greater than certain predefined threshold in the whole data set. At training stage the static mining process can be completed via offline, the pure data without containing intrusion data in some network environment is the data object with pertinence, the data is acquired by tcpdump, and pre-processed prior to mining. The result of static mining process is the normal behavior profile archive for delineating network or user normal behavior mode.

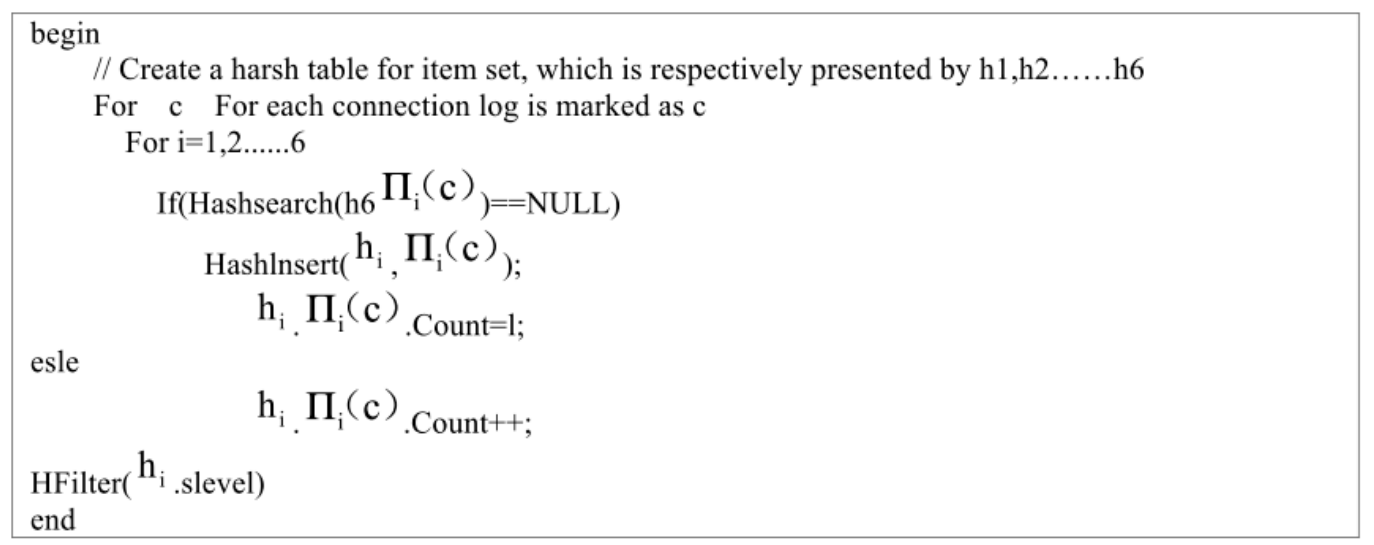

\subsection{Static Mining Algorithm}

Dynamic mining adopts slide window method to seek out suspected rule set via online incremental mining, next the detailed algorithm and false code description shall be analyzed. In order to seek out the suspected rule which is not consistent with normal behavior profile archive, In order to find out the suspected rule inconsistent with normal behavior profile archive, two operation stages of system shall be executed by dynamic mining process. During dynamic mining process it only needs to scan each connection log by one time, and the time window can also refer to volume window.

begin

The calculated hash table $\mathrm{h} 1, \mathrm{~h} 2$.....h $\mathrm{h} 6$ is adopted

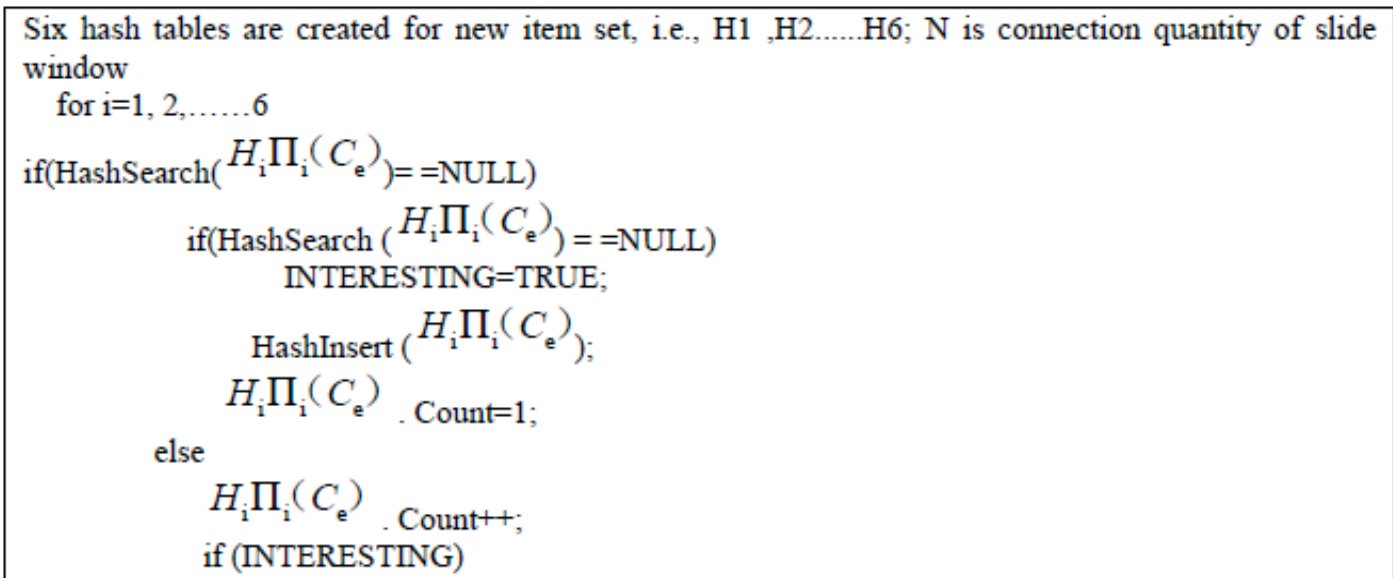




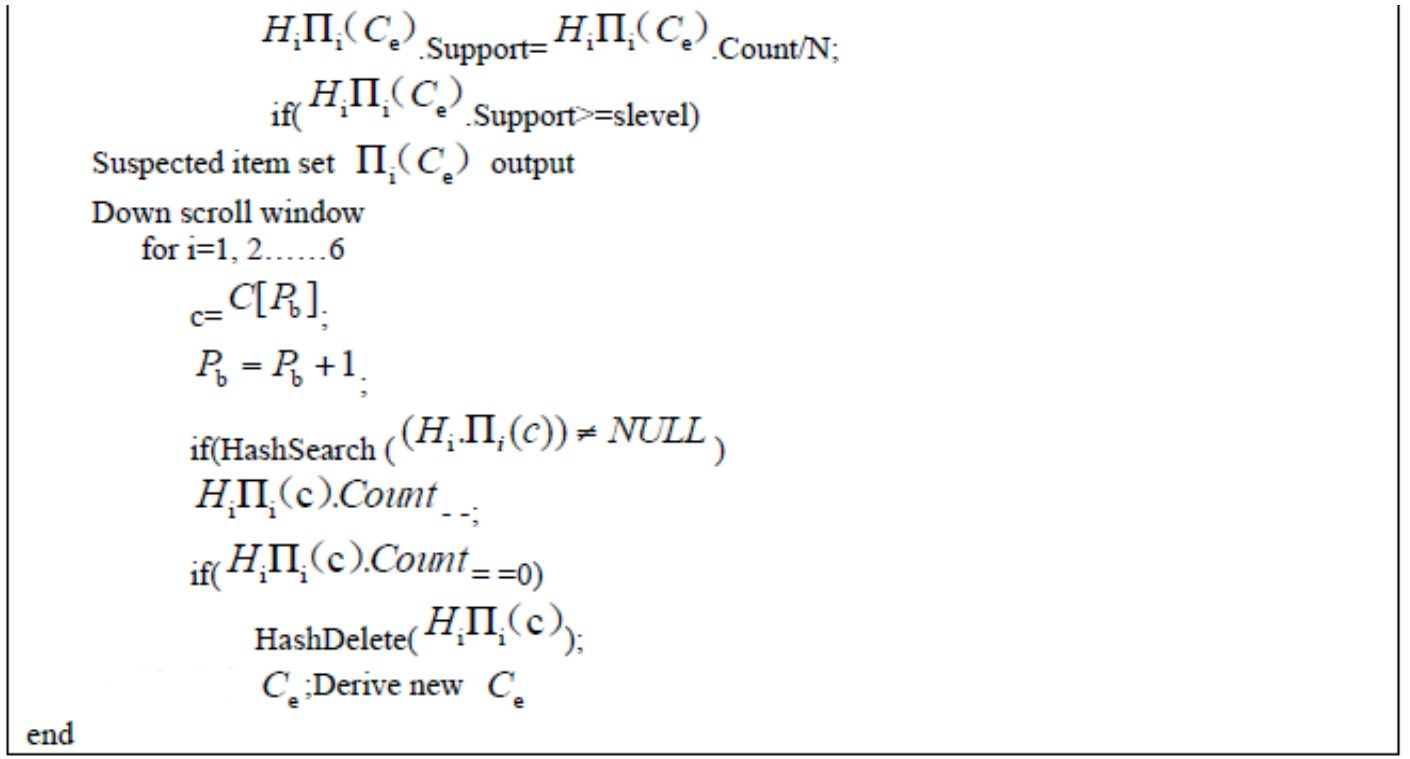

\subsection{Dynamic Mining Algorithm}

The single layer mining is to mine the data of pre-processing log of database without considering domain knowledge, it is only to seek out the association rule which is greater than certain threshold via the angle of data mining, it is most difficult and most important to seek out high frequent item set, therefore single layer mining is to seek out high frequent item set in the database, next, two conceptions of item set and association rule shall be alternatively used.

\author{
Src.IP, Dst.IP, \\ Src.IP, Dst. Port, \\ Src.IP, Dst.IP, Dst.Port, \\ Src.IP, Src.Port, Dst.IP, \\ Src.IP, Src.Port, Dst.Port, \\ Src.IP, Src.Port, Dst.IP, Dst.Port,
}

\subsection{Single Layer Mining}

The R (Ts, Src.IP, Src.Port, Dst.IP, Dst.Port, FLAG)

mode can be used to create higher level abstract of IP related attribute. Usually for B type address, the first byte of IP address host domain shall determine the subnet of the host, the first two bytes of IP address shall determine the network number of the host.

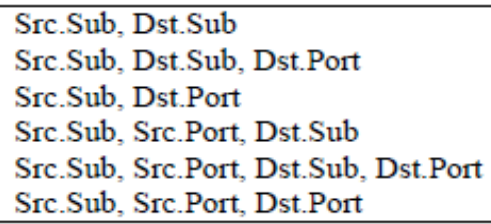

$\operatorname{Sub}\{\mathrm{IP}, \mathrm{Sub1}$, Sub2, Sub3, Sub4\} and Src.Sub $\neq$ Dst.Sub if Sub=IP

\subsection{Domain Layer Mining}

Table $\mathbf{1}$ is the detected attack quantity (PROBE. DOS, PASSWD and DIC), false report number, missing report number, when different support degrees are used in dynamic mining algorithm, it shall be used as rule quantity information inputted for decision tree.
Above test result shows that, decision tree plays an important role for reducing false report rate. Since attack type of test data once occurred in training data, decision tree after training shall have the knowledge of such attack type, and it is found that the decision tree misses four attack behaviors that should be detected, it is mainly due to overfitting determined by own limitation of decision tree. Naive Bayes: Naive $[9,10]$. 
Table 1. DARPA1998 test data result.

\begin{tabular}{|c|c|c|c|c|c|c|c|c|c|c|c|c|}
\hline \multirow{2}{*}{\multicolumn{2}{|c|}{$\begin{array}{c}\text { Week } \\
\text { Workday }\end{array}$}} & \multicolumn{2}{|c|}{$1^{\text {st }}$ week } & \multirow[b]{2}{*}{ Wed } & \multirow[b]{2}{*}{ Thu } & \multirow[b]{2}{*}{ Fri } & \multicolumn{2}{|c|}{$2^{\text {nd }}$ week } & \multirow[b]{2}{*}{ Wed } & \multirow[b]{2}{*}{ Thu } & \multirow[b]{2}{*}{ Fri } & \multirow[t]{2}{*}{ Total } \\
\hline & & Mon & Tue & & & & Mon & Tue & & & & \\
\hline \multirow{4}{*}{$\begin{array}{c}\text { \#rules By } \\
\text { MN }\end{array}$} & $\mathrm{Sp}=0.01$ & 1045 & 1438 & 1353 & 964 & 1060 & 1044 & 1352 & 1098 & 1224 & 991 & 11569 \\
\hline & $\mathrm{Sp}=0.05$ & 579 & 666 & 713 & 713 & 514 & 600 & 682 & 622 & 622 & 553 & 6264 \\
\hline & $\mathrm{Sp}=0.1$ & 477 & 552 & 553 & 456 & 445 & 539 & 524 & 548 & 537 & 488 & 5119 \\
\hline & $\mathrm{Sp}=0.2$ & 424 & 520 & 483 & 441 & 440 & 522 & 482 & 527 & 500 & 461 & 4800 \\
\hline \multirow{4}{*}{ \#rules By DT } & $\mathrm{Sp}=0.01$ & 7 & 11 & 5 & 60 & 17 & 81 & 16 & 7 & 18 & 17 & 239 \\
\hline & $\mathrm{Sp}=0.05$ & 7 & 11 & 5 & 46 & 17 & 81 & 13 & 7 & 17 & 16 & 220 \\
\hline & $\mathrm{Sp}=0.1$ & 7 & 11 & 5 & 46 & 12 & 80 & 10 & 7 & 17 & 13 & 208 \\
\hline & $\mathrm{Sp}=0.2$ & 7 & 10 & 5 & 46 & 10 & 80 & 9 & 6 & 14 & 12 & 199 \\
\hline \multirow{4}{*}{$\#$ fp } & $\mathrm{Sp}=0.01$ & 0 & 0 & 0 & 5 & 0 & 0 & 0 & 0 & 0 & 0 & 5 \\
\hline & $\mathrm{Sp}=0.05$ & 0 & 0 & 0 & 5 & 0 & 0 & 0 & 0 & 0 & 0 & 5 \\
\hline & $\mathrm{Sp}=0.1$ & 0 & 0 & 0 & 5 & 0 & 0 & 0 & 0 & 0 & 0 & 5 \\
\hline & $\mathrm{Sp}=0.2$ & 0 & 0 & 0 & 5 & 0 & 0 & 0 & 0 & 0 & 0 & 5 \\
\hline \multirow{4}{*}{ \#det } & $\mathrm{Sp}=0.01$ & 4 & 5 & 4 & 7 & 7 & 10 & 8 & 5 & 10 & 11 & 71 \\
\hline & $\mathrm{Sp}=0.05$ & 4 & 5 & 4 & 7 & 7 & 10 & 7 & 5 & 10 & 11 & 70 \\
\hline & $\mathrm{Sp}=0.1$ & 4 & 5 & 4 & 7 & 7 & 10 & 6 & 5 & 10 & 11 & 69 \\
\hline & $\mathrm{Sp}=0.2$ & 4 & 4 & 4 & 7 & 6 & 10 & 3 & 5 & 9 & 10 & 62 \\
\hline \multirow{4}{*}{$\#$ fn } & $\mathrm{Sp}=0.01$ & 2 & 0 & 0 & 0 & 0 & 1 & 1 & 3 & 1 & 0 & 8 \\
\hline & $\mathrm{Sp}=0.05$ & 2 & 0 & 0 & 0 & 0 & 1 & 2 & 3 & 1 & 0 & 9 \\
\hline & $\mathrm{Sp}=0.1$ & 2 & 0 & 0 & 0 & 0 & 1 & 2 & 3 & 1 & 0 & 8 \\
\hline & $\mathrm{Sp}=0.2$ & 2 & 1 & 0 & 0 & 0 & 1 & 2 & 3 & 1 & 1 & 10 \\
\hline
\end{tabular}

Table 2. DARPA1999 test data result.

\begin{tabular}{|c|c|c|c|c|c|c|c|c|c|c|c|c|}
\hline \multirow{2}{*}{\multicolumn{2}{|c|}{$\begin{array}{c}\text { Week } \\
\text { Workday }\end{array}$}} & \multicolumn{2}{|c|}{ 1st week } & \multirow[b]{2}{*}{ Wed } & \multirow[b]{2}{*}{ Thu } & \multirow[b]{2}{*}{ Fri } & \multicolumn{2}{|c|}{ 2nd week } & \multirow[b]{2}{*}{ Wed } & \multirow[b]{2}{*}{ Thu } & \multirow[b]{2}{*}{ Fri } & \multirow[t]{2}{*}{ Total } \\
\hline & & Mon & Tue & & & & Mon & Tue & & & & \\
\hline \multirow{4}{*}{$\begin{array}{c}\text { \#rules By } \\
\text { MN }\end{array}$} & $\mathrm{Sp}=0.01$ & 1320 & 1137 & 1452 & 1416 & 2003 & 1615 & 1596 & 1549 & 1511 & 1346 & 14945 \\
\hline & $\mathrm{Sp}=0.05$ & 866 & 750 & 1345 & 1263 & 1756 & 1245 & 1446 & 1375 & 1425 & 1156 & 12627 \\
\hline & $\mathrm{Sp}=0.1$ & 422 & 415 & 664 & 641 & 666 & 533 & 634 & 613 & 625 & 632 & 5845 \\
\hline & $\mathrm{Sp}=0.2$ & 333 & 368 & 595 & 595 & 617 & 388 & 530 & 600 & 525 & 496 & 5047 \\
\hline \multirow{4}{*}{ \#rules By DT } & $\mathrm{Sp}=0.01$ & 21 & 4 & 15 & 11 & 10 & 39 & 17 & 7 & 19 & 10 & 153 \\
\hline & $\mathrm{Sp}=0.05$ & 15 & 4 & 10 & 4 & 10 & 38 & 17 & 6 & 18 & 9 & 131 \\
\hline & $\mathrm{Sp}=0.1$ & 9 & 4 & 6 & 3 & 5 & 37 & 16 & 5 & 13 & 8 & 106 \\
\hline & $\mathrm{Sp}=0.2$ & 6 & 4 & 4 & 3 & 5 & 36 & 15 & 5 & 10 & 7 & 95 \\
\hline \multirow{2}{*}{$\# \mathrm{fp}$} & $\mathrm{Sp}=0.01$ & 0 & 0 & 0 & 2 & 1 & 2 & 1 & 1 & 2 & 1 & 10 \\
\hline & $\mathrm{Sp}=0.0$ & 0 & 0 & 0 & 2 & 1 & 2 & 1 & 1 & 2 & 1 & 10 \\
\hline
\end{tabular}


Table 3. DARPA attack data information.

\begin{tabular}{|c|c|c|c|c|}
\hline \multirow{2}{*}{ Year } & Training data & & Test data & Attack type \\
\cline { 2 - 5 } & Attack type & All attack quantity & 24 & 65 \\
\hline \hline DARPA1998 & 11 & 114 & 27 & 106 \\
\hline DARPA1999 & 7 & 18 & 27 & \\
\hline
\end{tabular}

Table 4. Training and test configuration scheme.

\begin{tabular}{|c|c|c|}
\hline Data set & Training data & Test data \\
\hline \hline Data set 1 & DARPA1998 & DARPA1998 \\
\hline Data set 2 & DARPA1999 & DARPA1999 \\
\hline Data set 3 & DARPA1999, DARPA1999 \\
\hline
\end{tabular}

Table 5. Attack cluster.

\begin{tabular}{|c|c|c|c|}
\hline $\mathbf{1}$ & $\mathbf{2}$ & $\mathbf{3}$ & $\mathbf{4}$ \\
\hline \hline Neptune & Satan & Nmap & Smurf \\
\hline Back & Portsweep & Ipsweep & \\
\hline Dict.guest & Saint & & \\
\hline Pod.teardrop & & & \\
\hline Mailbomb & & & \\
\hline Udpstorm & & & \\
\hline Snmpguess & & & \\
\hline Snmpgetattack & & & \\
\hline
\end{tabular}

Table 6. Attack data distribution in DARPA98 training.

\begin{tabular}{|c|c|c|c|c|c|c|c|c|c|c|c|}
\hline Cluster & 1 & & & & & & 2 & & 3 & & 4 \\
\hline Name & Netptune & Back & Dic $t$ & Guest & Po d & Teardro $p$ & Sata $n$ & Portsweep & Nam $p$ & Ipsweep & smurf \\
\hline
\end{tabular}

Table 7. Attack data distribution of DARPA98 test.

\begin{tabular}{|c|c|c|c|c|c|c|c|c|c|}
\hline Cluster & 1 & & & & & & & & \\
\hline Name & Netptun e & Back & Dic $t$ & Guest & Po d & Teardro $\mathrm{p}$ & Mailbo x & Udpstro m & Land \\
\hline
\end{tabular}




\begin{tabular}{|c|c|c|c|c|c|c|c|c|c|}
\hline Cluster & $\mathbf{1}$ & & $\mathbf{2}$ & & $\mathbf{3}$ & & $\mathbf{4}$ \\
\hline \hline Name & snmpgue ss & snmpget & satan & Porswe ep & saint & nma p & ipsweep & smurf & Mscan \\
\hline Attack & 2 & 6 & 2 & 5 & 2 & 4 & 3 & 8 & 1 \\
\hline
\end{tabular}

Bayes classifier bases one of following simplehypothesis: mutualconditi (on) on of each attribute value is independent. Bayes theorem is used to predict a possibility that an unknown sample belongs to each class, the class with biggest possibility is selected as final class of the sample. Detailed given instance $\mathrm{E}=\mathrm{XX}$...... $\mathrm{X}$, herein $\mathrm{E}$ has $\mathrm{m} 12 \mathrm{~m}$ attributes, Naive $B($, )ayes is to classify $E$ into $P(C \mid E)$ with highest condition probability of $\mathrm{K} \mathrm{CK}$. According to independency hypothesis:

$$
\begin{aligned}
& P\left(C_{k} \mid E\right)=\frac{P\left(E \mid C_{k}\right)}{P(E)} \\
& =\frac{P\left(X_{1} \mid C_{k}\right) P\left(X_{2} \mid C_{k}\right) \mathrm{K} P\left(X_{n} \mid C_{k}\right) P\left(C_{k}\right)}{P(E)}
\end{aligned}
$$

For dispersed variable, $P\left(X \mid C_{k}\right) \mathrm{P}(\mathrm{X} \mid \mathrm{CK})$ is estimated as I per sample frequency in training data.

Table $\mathbf{2}$ is the attack type and attack instance information of DARPA data set. It is apparent that DARPA1998 training data has more attack types and attack instances than DARPA1999 training data, furthermore, difference of attack type and attack instance of DARPA1999 is far greater than that of DARPA1998 data set.

For detection of unknown type attack, the biggest advantage of anomaly detection method is to detect new attack type data, nevertheless at present, the performance of intrusion monitoring system for detecting new attack type is not ideal.

Tables 6 and 7 test results give the distribution situation of every cluster attack data of DARPA1998 training data and test data.

In this test, the false code of NAIDS system to core algorithm is given, a large number of tests on DARPA 1998. 1999 intrusion detection evaluation data set demonstrate the effectiveness and science of the method of this paper. In order to enable the system process massive network auditing data, slide window technology is firstly proposed in mining module.

\section{GENERALIZATION}

NAIDS is the first anomaly detection system based on data mining technology, association rule mining algorithm is adopted to establish system and user normal behavior profile archive under network environment, and seek out suspected mode of network traffic volume data, slide window technology based dynamic mining algorithm is proposed to realize online incremental mining, to in some extent ensure real time performance of NAIDS system, aiming at anomaly behavior inconsistent with normal behavior profile archive, the system can provide the capability of judging anomaly source by tracing back to auditing data, it can be in favor of system safety staffs' participation, normal and anomaly deviation of learning behavior profile archive can adopt monitoring type classification engine. The test data proves that it can greatly reduce false report rate of system, in order to enable NAIDS really have anomaly detection, decision tree is improved to seek out new attack type, absolute support degree conception is proposed in combination with domain issue, the test proves that it can greatly improve system performance.

\section{CONFLICT OF INTEREST}

The author confirms that this article content has no conflict of interest.

\section{ACKNOWLEDGEMENTS}

This work was supported by the soft science study plan project of Ningbo (2011A1014).

\section{REFERENCES}

[1] R. Agrwaal and R.Sriknat, "Fast algorithm for mining association rules", in Proe. 20Int. Conf. Verye Date Large DatBaases, Snatigao Chile, pp. 487-499, Sep. 1994.

[2] J. R. Quinlna, "Induction of decision trees", Machine Lemaing, vol. 1, pp. 81-106, 1986.

[3] W. W. Cohen, "Fast effective rule induction", in Machine Lemaing: Pore of the12 international Conference, Lake Tahoe, Califonria, 1995.

[4] R. Ng and J. Hna, "Efficient and effective setring method for spatial data mining", in Proe. 20Int. Conf Verye Large DatBaases, Snatigao Chile, Sep. 1994, pp. 144-155.

[5] T. Zhang, R. Rmakarishnna, and M. L. .Birch, "An effieient data clustering method for veyr large databases", in Proe.ACM-SIGMODInt.Conf Mnagaement of Data, Montreal, Canada, June 1996, pp. 103-144.

[6] R. Agarwal and R. Sriknat, "Mining Sequeniial Pattenr", in Proe. Int. Conf. Data Engineering, TaiPei Taiwan, May 1995, pp. 3-14.

[7] W. Lee and S. Stolfo, "Data mining approaches for intursion detection", in San Antonio. Tx: Proe.7 USENIX Security Symposium, 1998, pp. 79-94.

[8] E. Eskin, A. Arnold, and M. Prerau, "A Geometric framework for anomaly detection: detecting intrusions in unabled data", in Data mining Application, Kluwer, 2002. 
[9] H. J. George and P. Langley, "Estimating continuous distributions in Bayesian classifiers", in proceedings of Conference on Uncertainty in Artificial Intelligence, San Mateo, 1995.

Received: July 02, 2014

Revised: September 10, 2014

Accepted: November 15, 2014

(C) Jing Xu; Licensee Bentham Open.

This is an open access article licensed under the terms of the Creative Commons Attribution Non-Commercial License (http://creativecommons.org/-licenses/ by-nc/3.0/) which permits unrestricted, non-commercial use, distribution and reproduction in any medium, provided the work is properly cited. 